\title{
Editorial
}

\section{The Case for Flu Vaccination}

Olukayode A. Akinlaja, MD, MBA, FACOG*

Department of Obstetrics \& Gynecology, University of Tennessee College of Medicine, Chattanooga, USA

\section{"Corresponding author \\ Olukayode A.Akinlaja, MD, MBA, FACOG}

Assistant Professor, Department of Obstetrics \& Gynecology, University of Tennessee College of Medicine, Chattanooga, USA;

E-mail: Olukayode.Akinlaja@erlanger.org

\section{Article Information}

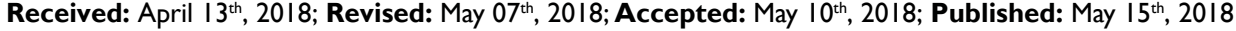

\section{Cite this article}

Akinlaja OA. The case for flu vaccination. Vaccin Res Open J. 2018; 3(I): e4-e5. doi: I0.I7I40/VROJ-3-e006

$\mathrm{I}^{\mathrm{r}}$ nfluenza or flu vaccine encompasses the seasonal vaccination that protects against infection by the influenza viruses. ${ }^{1}$ It's generally available in two forms; the inactive form administered as shots or intramuscular injections and the weakened live viral forms sprayed intranasally.

The flu vaccination is generally safe and listed among the WHO list of Essential Medicines. ${ }^{2}$ It is currently the best way to protect the populace from the flu, thereby preventing it's spread and both the United States Center for Disease Control and Prevention (CDC) and the World Health Organization (WHO) recommend yearly vaccination for nearly all people beyond six months of age, especially if in high risk groups such as pregnant, healthcare workers, elderly, those with other healthcare conditions and children between six months and five years of age. ${ }^{3,4}$

The influenza vaccination was initiated in the 1930's but achieved large scale availability in the United States in $1945^{5}$ and even though their effectiveness varies from year to year, it has been established by the CDC that they do reduce the incidence of influenza related sickness, medical visits, hospitalizations and deaths. ${ }^{6}$

On vaccination, it takes about two weeks to form protective antibodies ${ }^{7}$ and as of 2015, each influenza vaccine dose cost less than $\$ 25$ in the United States and very much less in most developing nations. ${ }^{8}$

The flu vaccination has been rarely linked to the development of Guillain-Barré syndrome ${ }^{9}$ but established side effects ranging from runny nose, sore throat, muscle pain and fever to rare allergic reaction are relatively mild when compared to the severe effects of the annual influenza epidemic such as hospitalizations and death. ${ }^{1,7}$

The live attenuated version works by introducing an infection in the nasal passages while the inactivated form is based on an immune response to the antigens present on the inactivated virus and only the inactive version should be administered to pregnant women to assist in inducing protection of both mother and child from the effects of an influenza infection thereby increasing her chances of a successful full term pregnancy. ${ }^{10,11}$

Vaccine administration should be avoided in those with severe allergies to prior versions of the influenza vaccine and also since eggs are typically used in the processing of the flu vaccines, caution should be taken in those with allergy to eggs and history of Guillain-Barre syndrome. ${ }^{12,13}$

Egg allergy cases are now being circumvented by growing the vaccine in insect cells rather than eggs ${ }^{14}$ and the Global Advisory Committee on Vaccine Safety of the World Health Organization has found no evidence to be fearful of toxicity from the Mercury based preservative, thimerosal. ${ }^{15}$ Nevertheless, thimerosal-free versions of flu vaccines are available in developed countries.

The need for vaccination against influenza in high-risk groups cannot be overemphasized and it should be routinely offered seasonally as the risk-benefit ratio is definitely in support of vaccination.

\section{REFERENCES}

1. Vaccines against influenza WHO position paper. Wkly Epidemiol Rec. 2012; 87(47): 461-476.

2. WHO model list of essential medicines (19th List). World Health Organization Web site. http://www.who.int/medicines/ publications/essentialmedicines/EML_2015_FINAL_amended_ NOV2015.pdf?ua=1 Published April, 2015. Amended November, 2015. Accessed March 30, 2018.

3. WHO Should Get Vaccinated Against Influenza. U.S. Centers for Disease Control and Prevention Web site. https://www.cdc. gov/flu/protect/whoshouldvax.htm Published November 4, 2015. Accessed March 30, 2018. 
4. Influenza (Seasonal) Fact sheet. World Health Organization. Web site. http://www.who.int/en/news-room/fact-sheets/detail/ influenza-(seasonal). Published November 2016. Accessed March 31, 2018.

5. Nunnally, Bk, Turula, VE, Sitrin, RD, eds. Vaccine Analysis: Strategies, Principles and Control. Heidelberger, Berlin, Germany: Springer. 2014.

6. Estimated Influenza Illnesses, Medical Visits, Hospitalizations, and Deaths Averted by Vaccination in the United States. Centers for Disease Control and Prevention. Web site. https://www.cdc. gov/flu/about/disease/2015-16.htm. Published December 9, 2016. Accessed May 7, 2018.

7. Key Facts About Seasonal Flu Vaccine. U.S. Centers for Disease Control and Prevention. Web site. https://www.cdc.gov/flu/protect/keyfacts.htm. Accessed March 30, 2018.

8. Hamilton RJ. Tarascon Pocket Pharmacopoeia 2015. Deluxe LabCoat Edition. Burlington, Massachusetts, USA; Jones \& Bartlett Learning. 2015.

9. Stowe J, Andrews N, Wise L, Miller E. Investigation of the temporal association of Guillian-Barre Syndrome with influenza vaccine and influenza like illness using the United Kingdom General Practice Research Database. Am J Epidemiol. 2009; 169(3): 382-388. doi: $10.1093 /$ aje/kwn310
10. Product Monograph: Flumist, Astrazeneca Canada Inc., 2011. Web site. https://www.astrazeneca.ca/content/dam/az-ca/downloads/productinformation/flumist-qlaiv-product-monograph-en. pdf. Published. April 19, 2018. Accessed May 7, 2018.

11. Fell DB, Sprague AE, Liu N, et al. H1N1 influenza vaccination during pregnancy and fetal and neonatal outcomes. Am J Public Health. 2012; 102(6): e33-e40. doi: 10.2105/AJPH.2011.300606.

12. Inactivated Influenza Vaccine 2007-2008 - What You Need To Know. U.S. Centers for Disease Control and Prevention. Web site. https://web.archive.org/web/20071120001659/http://www.cdc. gov/vaccines/pubs/vis/downloads/vis-flu.pdf. Retrieved March 31, 2018. Accessed May 7, 2018.

13. Seasonal Flu Shot - Seasonal Influenza (Flu). U.S. Centers for Disease Control and Prevention. Retrieved March 31, 2018.Accessed May 7, 2018.

14. Pollack A. Rapidly Produced Flu Vaccine Wins F.D.A. Approval. The New York Times. January 16, 2013. https://www. nytimes.com/2013/01/17/business/flublok-a-flu-vaccine-wins-fdaapproval.html. Accessed May 7, 2018.

15. Thiomersal and Vaccines. World Health Organization. Web site. http://www.who.int/vaccine_safety/committee/en/. Published March 31, 2018. Accessed May 7, 2018. 